formed in the north, and continued to shine with more or less brilliancy for some time. The arc appeared to be a double one, by the presence of a dark band running longitudinally through it. Occasional streamers of equally pure white light ran upwards from either end of the bow. The moon was only a day old, but the old landscape was lighted up as if by the full moon; and the effect of Kyle Akin lighthouse, the numerous surrounding islands, and the still sea between, was a true thing of beauty, forming as it did a quiet contrast to the more brilliant but restless forms of auroræe generally seen. I particularly noticed a somewhat misty and foggy look about the brilliant arc, giving it almost a solid appearance. The space of sky between the horizon and the lower edge of the arc was of a deep indigo colour, probably the effect of contrast.

I regretted I had no spectroscope with me, as it would have been a fine opportunity to test the spectrum of an aurora of pure white light. I had a strong impression that the bow was near to the earth, and almost thought that the eastern end, and some fleecy clouds in which it was involved, were between myself and the peaks of some distant mountains. The eye is, however, deceptive in such cases, though instances are not wanting of aurorre close to the earth's surface. I shall be glad to know if other observations of this aurora were made.

Nairn, N.B., Oct. 3

\section{The Cry of the Frog}

THF fact that the common frog (Rana temporaria) is capable of crying out lustily when he feels himself in danger, does not seem to have been frequently remarked. In my small walled garden there is a common frog who is persecuted by three cats. His residence is a heap of slates at the foot of an ivied wall, and here he is safe. But if he ventures far abroad his tormentors soon espy him, and though they seem nearly as much terrified as himself, they cannot resist the temptation to touch him with their paws. He immediately opens his mouth and utters a prolonged crý, which appears to be very surprising to the cats, who draw back for 2 few moments, and then pat him again, apparentiy out of mere curiosity, to be again scared by the same unusual sound. This sound is a shrill and rather sibilant wail, like the note of a sinall penny trumpet or the cry of a new-born infant. There can be no mistake about it, as I have repeatedly touched the frog with my own hand after driving the cats away, and the same cry has immediately followed, the lower jaw being dropped so that the mouth stands open about a quarter of an inch at the tip.

Leicester, Sept. 26

F. T. MOT'T

\section{The Woolwich Aëronautical Experiment} II.

IN order to ciscover the laws of the vertical motion, we must suppose that the balloon is resting in perfect equilibrium. when on land; which means that the ascending power of the gas enclosed in the balloon is just equal to the weight of the canvas, netting, grapnel, ballast, passengers, \&c. Under these circumstances the iallonn will not ascend by itself, but it will with all the weight of the sand which may be thrown overboard, if a certain space is left for dilatation and the balloon is not quite full when resting on land. If the rolume is $\mathrm{V}$ at the surface of earth, it will be $\frac{V H}{h}$ at an altitude where barometric pressure is $h$, being

$I$ at departure. When the balloon is quite full, gas escapes by the lower part under the shape of a whitish steam. If $v$ is the additional volume which can be filled by dilatation, that phenomenon will take place at an altitude where the pressure is $h$ given by the equation $\frac{V H}{V+v}:$ :

We suppose that the height $h$ is never to be attained, and in fact it is desirable for the aëronauts to limit their altitude before starting, and not to fill their balloon with a gas which they are obliged to throw away by the valve or to see escaping by the appenaice at some risk of their own safety; one of the greatest advantages of the vertical fan being to limit at will the ascent, as will be shown.

In our calculations we suppose that the canvas is not losing gas, that the sun is not affecting the balloon, and that no water is falling upon it, or no cloud concealing the sun. All these changes of temperature can be made the subject of special calculations, and the real motion of the aërostatic globe is the mean between all the different circumstances of the atmøsphere.

If a balloon starts in an homogeneous air because a weight $\not D$ of sand was thrown overboard, $P$ being the weight of the air displaced by the balloon when resting on land, the motive power is $g^{\prime}=\frac{g p}{P+p}$ and the laws of the motions of an Attwood machine are perfectly applicable to it.

The elevation takes place with an increased velocity up to the moment where the resistance; of the air is $=$ to $g^{\prime}$. Consequently,

$$
K v^{2}=\frac{p g}{P+p}
$$

$K$ being a certain coefficient which tepends on the form of the balloon, its diameter, its netting, and the density of the air. $K$ diminishes as the altitude increases, but the diameter of the balloon enlarges gradually to its utmost. As the law of diminution of pressure is not known, we are obliged to suppose $K=$ constant.

If we suppose a balloon of 60,000 cubic feet holding 50,000 cubic feet of gas when resting on the ground, the balloon can reach without losing gas (except by the loss through the canves, which we suppose to be perfectly gas-tight) to a level where $h^{\prime}=\frac{5 h}{6}=$ about 6,000 feet in round numbers. Under these circumstances the weight of the balloon when resting on land may be supposed to be about 3,300 pounds.

If we suppose 20 lbs. of sand are thrown overboard in ascending, the motive power will be $\frac{g}{\mathrm{I} 5}$. The uniform motion will be $K v^{2}=\frac{g}{115}$.

Under these circumstances, as far as my knowledge goes, it is $4 \mathrm{ft}$. per second. If we suppose $g=32$ feet.

$$
K \pi^{2}=16 K=\frac{32}{115} \text { and } K=\frac{32}{115 \times 16}=\frac{2}{115}
$$

If a static effort of 20 lbs. in the vertical direction can be produced by the working of the vertical fan, it is easy to understand that the ascent can be stopped before the balloon has reached the level where the gas is beginning to escape by working in the proper direction for it. That effort is not too much for two men working on a fan which is suitably constructed.

The same thing can be said as to the descent of the balioon, but $K$ is much larger, as the shape of the lower part is not so well suited tor moving in the air as the upper half. With appendice, netting, ropes, and car, it exerts a resistance which is much larger and may be compared with the force exerted by a parachute descending in the air. The difference is very great, as I observed several times in my ascents that it was difficult to give the balloon a descending impulsion towards the land. I should not wonder if it was partly the cause of the resistance felt by Mr. Bowdler when moving his fan in the direction where it ought to liave caused the balloon to descend; at least such is the opinion that $I$ am in position to hold from the concise and imperfect narrative I fo:nd in the public papers.

\section{W. DE FONVIELIE}

\section{Is the Rabbit Indigenous?}

WovLn you permit me, through the medium of NATURE, to ask on what grounds the rabbit is considered not indigenous in this country? The best authorities on British and Germai Mammalia seem agreed that it is a native of the Mediterranean basin. On what facts or writings is this opinion based, and at what time was it introduced into Great Britain? I am very anxious to determine whether the above statements are founded on authentic documents or writings, or are merely suppositions which cannot be asserted with certainty.

Sept. 30

\section{THE SOCIAL SCIENCE CONGRESS}

THE friends of social science have had a most successful meeting this year at Glasgow, and in the various addresses and papers there has been afforded ample evidence that the importance of the introduction of more scientific knowledge into the heads and daily life of the people is becoming more and more widely acknowledged.

In the Health Section, Dr. Lyon Playfair in his address; 